\title{
CHIARITÉ
}

UNIVERSITÄTSMEDIZIN BERLIN

\section{Psychosoziale Beratung in einem gemeindepsychiatrischen Zentrum - eine qualitative Studie über die Erfahrungen der Nutzer}

Julian Schwarz, Anne Berghöfer, Burkhart Brückner, Thomas Birker, Barbara Stöckigt

\section{Document type}

Postprint (accepted version)

This version is available at

https://doi.org/10.17169/refubium-32724

\section{Citation details}

Schwarz J, Berghöfer A, Brückner B, Birker T, Stöckigt B. Psychosoziale Beratung in einem gemeindepsychiatrischen Zentrum - eine qualitative Studie über die Erfahrungen der Nutzer. PPmP - Psychotherapie - Psychosomatik - Medizinische Psychologie. Georg Thieme Verlag KG; 2018. p. 167-175. DOI: 10.1055/a-0594-2616

\section{Terms of use}

All rights reserved. This document is intended solely for personal, non-commercial use. 


\section{Psychosoziale Beratung in einem gemeindepsychiatrischen Zentrum - eine qualitative Studie über die Erfahrungen der Nutzer}

Users' experiences with psychosocial counselling services in a community mental health centre.

Julian Schwarz ${ }^{1}$, Anne Berghöfer ${ }^{2}$, Burkhart Brückner ${ }^{3}$, Thomas Birker ${ }^{4}$, Barbara Stöckigt ${ }^{2}$

1) Hochschulklinik für Psychiatrie und Psychotherapie der Medizinischen Hochschule Brandenburg, Immanuel Klinik Rüdersdorf

Dr. med. Julian Schwarz

Seebad $82 / 83$

15562 Rüdersdorf bei Berlin

julian.schwarz@mhb-fontane.de

2) Institut für Sozialmedizin, Epidemiologie und Gesundheitsökonomie, Charité - Universitätsmedizin Berlin, Berlin

3) Fachbereich Sozialwesen, Hochschule Niederrhein University of Applied Sciences, Mönchengladbach

4) Klinik für Psychiatrie, Psychotherapie und Psychosomatik, Westküstenkliniken Brunsbüttel und Heide gGmbH, Heide 


\section{Zusammenfassung}

Ziel: Analyse der Erfahrungen von Nutzern einer innovativen, rechtskreisübergreifenden psychosozialen Beratungsstelle. Methode: Neun Einzelinterviews wurden qualitativ-inhaltsanalytisch ausgewertet. Ergebnisse: Das Angebot stärkte psychosoziale Ressourcen und die Autonomie der Nutzer. Positiv wurde die aufsuchende Beratung bewertet, kritisiert wurde die begrenzte Sichtbarkeit des Angebots. Schlussfolgerungen: Eine rechtskreisübergreifende Beratungsstelle kann Hilfesuchende in Krisen auffangen und passgenau in gemeindepsychiatrische oder psychosoziale Angebote vermitteln.

\section{Abstract}

Objective: Analysis of users experiences with a low-threshold psychosocial counselling and case management service across separate sectors (e. g. outpatient, inpatient) and legal provisions (e. g. V and XII Books of Social Code). Methods: Nine semi-structured interviews were conducted and analysed using content analysis. Results: Several aspects of the users' experiences with counselling services have contributed towards their satisfaction with the service. The importance of short waiting times and the on-call telephone service as well as sufficient time taken for consultations, the availability of outreach counselling and the quality of the relationship with the counsellor were all highlighted. Potential for improvement was seen in the visibility of the service in the community. Conclusion: The investigated counselling and case management service can help to provide a low-threshold crisis intervention and could place users in more custom-fit community integration services.

\section{Schlüsselwörter}

- Psychosoziale Beratung

- Erfahrungen der Nutzer

- Krisenintervention

- Patientenzufriedenheit

- Aufsuchende Dienste

\section{Keywords}

- psychosocial counselling

- users' experiences

- crisis intervention

- patient satisfaction

- outreaching teams 


\section{Einleitung}

Eines der gegenwärtig größten Hindernisse für eine angemessene psychosoziale Versorgung in Deutschland ist ihre Fragmentierung in verschiedene Sozialgesetzbücher (SGB). Maßgeblich sind Leistungen zur medizinischen Behandlung (SGB V) und zur Eingliederung von Menschen mit seelischen Beeinträchtigungen (SGB XII). Durch diese Trennung kann die Zusammenarbeit zwischen medizinischen und sozialfürsorgerischen Diensten nur äußert begrenzt stattfinden. Dies wiederum behindert die patientenzentrierte und bedürfnisorientierte Versorgung in der Gemeinde.

Zwar wurde mit dem Bundesteilhabegesetz die Stärkung ambulanter Unterstützungsformen beschlossen [1] und die Einführung der stationsäquivalenten psychiatrischen Behandlung ( $\$ 115 d$ SGB V) verspricht eine sektorenübergreifende Akutversorgung im häuslichen Umfeld [2], unklar bleibt jedoch, wie die einzelnen Behandlungs- und Teilhabeleistungen koordiniert werden könnten.

In diesem Zusammenhang haben Steinhart und Wienberg ein Modell vorgestellt, dessen Implementierung hilfreich sein könnte, um die Problematik zu überwinden [3,4]. Zentrale Instanz dieses Funktionalen Basismodells für die gemeindepsychiatrische Versorgung schwer psychisch kranker Menschen ist eine Koordinations- und Beratungsstelle, die sowohl (mobile) Behandlungs- als auch Teilhabeleistungen vorsieht. In Heide, Schleswig-Holstein, wurde ein Gemeindepsychiatrisches Zentrum (GPZ) gegründet, das seit dem 1. Januar 2014 die Kernelemente und -funktionen eines solchen Modells erfüllt (vgl. Abb. 1, Tab. 1):

Das GPZ bietet für den ca. 130.000 Einwohner großen Kreis Dithmarschen eine zentral angesiedelte psychosoziale Beratungsstelle, die rund um die Uhr und auch aufsuchend tätig ist. Neben Unterstützung in psychosozialen Krisen wird eine anbieterneutrale Beratung und Koordination für gesundheitsbezogene (SGB V) und Leistungen der Eingliederungshilfe (SGB XII) angeboten. Durch diese rechtskreisübergreifende Funktion sollen insbesondere Nutzer mit komplexen Hilfebedarfen möglichst bedürfnisorientiert und aus einer Hand versorgt werden. Durch die präventive Ausrichtung richtet sich das Angebot auch an Personen, die erstmalig Kontakt zum Hilfesystem aufnehmen. So soll versucht werden, möglichst frühzeitig in niederschwellige und komplementäre Leistungsangebote zu vermitteln, um einer „Psychiatrisierung“ vorzubeugen und die verfrühte Aufnahme in ressourcenaufwändige stationäre Behandlungs- und Wohnformen zu verhindern.

Neben den fachlichen Vorgaben des Basismodells, die das GPZ einlöst, stellt sich die Frage, inwiefern die Beratung auch den Bedürfnissen ihrer Nutzer entspricht und wie zufrieden diese mit der Beratung sind [5]. 
Tab. 1 Implementierung der Kernelemente und -funktionen der psychosozialen Versorgung im Landkreis Dithmarschen.

\begin{tabular}{|c|c|}
\hline Kernelemente und -funktionen & Implementierung vor Ort \\
\hline Steuerungsfunktion & $\begin{array}{l}\text { Zentrales Element ist die „GPZ-Auftragsrunde“, ein wöchent- } \\
\text { lich tagendes Gremium (aus Mitarbeitern des WKK und des } \\
\text { Fachdienstes EGH, Kreis Dithmarschen) zur anbieterneutralen, } \\
\text { bedürfnisorientierten Ermittlung und Koordination von Basis- } \\
\text { und Komplexleistungen in den Bereichen Behandlung und Teil- } \\
\text { habe. }\end{array}$ \\
\hline Beratung & \multirow{3}{*}{$\begin{array}{l}\text { Durchgeführt von Mitarbeitern des GPZ (in Personalunion von } \\
\text { WKK und Fachdienst EGH), kann bis zu dreimal in Folge (auch } \\
\text { aufsuchend) von Patienten bzw. Klienten in Anspruch genom- } \\
\text { men werden. }\end{array}$} \\
\hline $\begin{array}{l}\text { Prävention-, Behandlungs- und Ge- } \\
\text { nesungsplanung }\end{array}$ & \\
\hline $\begin{array}{l}\text { Rehabilitations- und Teilhabepla- } \\
\text { nung }\end{array}$ & \\
\hline $\begin{array}{l}\text { Mobile multiprofessionelle Be- } \\
\text { handlung }\end{array}$ & $\begin{array}{l}\text { Sektorübergreifend tätige Teams des WKK, Standort Heide im } \\
\text { Rahmen des psychiatrischen Modellvorhabens nach } § 64 b \text { SGB } \\
\text { V: }\end{array}$ \\
\hline Komplexe ambulante Behandlung & Home Treatment (kurzdauernde Krisenversorgung) \\
\hline Nachgehende Intensivbehandlung & Assertive Community Treatment (längerfristige Behandlung) \\
\hline Komplexe intensive Behandlung & $\begin{array}{l}\text { (Teil-) stationäre Behandlung des WKK, Standorte Heide und } \\
\text { Brunsbüttel }\end{array}$ \\
\hline (Akut-) Psychotherapie & Angeboten z.B. vom MVZ Brunsbüttel und Heide (Teil des WKK) \\
\hline $\begin{array}{l}\text { Mobile multiprofessionelle Unter- } \\
\text { stützung }\end{array}$ & $\begin{array}{l}\text { Ambulant und stationär tätige Mitarbeiter unterschiedlicher } \\
\text { EGH-Leistungsanbieter, z.B. der Brücke Schleswig-Holstein } \\
\text { gGmbH mit verschiedenen Standorten in Dithmarschen }\end{array}$ \\
\hline Soziale Teilhabe & $\begin{array}{l}\text { Selbsthilfegruppe Alkoholsucht (AWO Heide), Begegnungs- } \\
\text { stätte „Kiek-In“ (Heide), Förderverein Nervennahrung e.V., Of- } \\
\text { fene Sprechstunde für Suchtkranke (Diakonisches Werk Dith- } \\
\text { marschen), Niederschwellige Begegnungsstätte (Brücke Dith- } \\
\text { marschen e.V.), Schuldnerberatung (AWO Heide und Lichtblick } \\
\text { Brunsbüttel) }\end{array}$ \\
\hline Teilhabe Wohnen & $\begin{array}{l}\text { Stationäre und ambulante Wohnformen unterschiedlicher } \\
\text { EGH-Leistungsanbieter, z.B. der Brücke Schleswig-Holstein } \\
\text { gGmbH mit verschiedenen Standorten in Dithmarschen }\end{array}$ \\
\hline Teilhabe Arbeit & WfbM Stiftung Mensch, Meldorf \\
\hline
\end{tabular}

GPZ = Gemeindepsychiatrisches Zentrum, WKK = Westküstenklinikum, EGH = Eingliederungshilfe, WfbM = Werkstatt für behinderte Menschen 


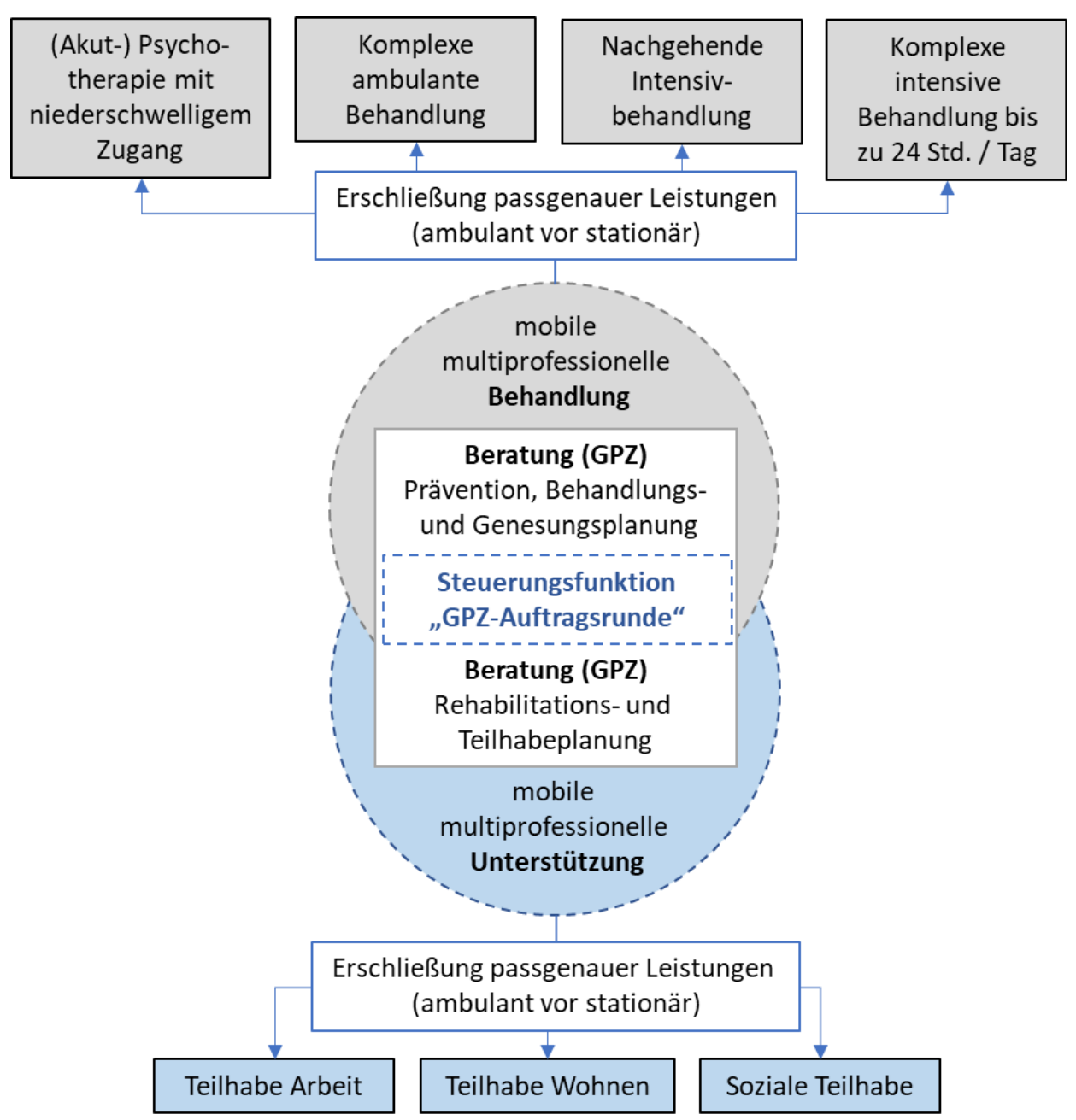

Abb. 1 Kernelemente und Funktionen der psychosozialen Versorgung im Landkreis Dithmarschen, adaptiert nach Steinhart \& Wienberg $(2016,2017)$. Blau hinterlegte Elemente sind überwiegend über SGB XII (Eingliederungshilfe), grau hinterlegte über SGB V (Krankenkasse) abgedeckt.

Die Zufriedenheit von Patienten wird in der Versorgungsforschung üblicherweise mit standardisierten Messgrößen, die auf Patienteneinschätzungen basieren, ermittelt [6,7]. Doch das Konstrukt „Patientenzufriedenheit" ist durchaus problematisch [8,9]: Einerseits hängt die Zufriedenheit nicht nur von behandlungsabhängigen Faktoren, sondern auch von zahlreichen Patientenmerkmalen ab $[9,10]$. Andererseits gibt es nur wenig Instrumente, die neben der Globalzufriedenheit auch die zugrundeliegenden Einflussfaktoren erfassen $[11,12]$.

Ziel dieser Studie ist es, die Ursachen der (Un-)Zufriedenheit der Nutzer zu explorieren, um mögliche Verbesserungen für die Praxis abzuleiten. Eine Methode, die diesen Anforderungen entspricht, ist die qualitative Erhebung von Patientenerfahrungen [13-16]. Hieraus soll schließlich ein differenziertes Zufriedenheitsverständnis abgeleitet werden. 
Die vorliegende Arbeit ist Bestandteil einer dreiteiligen Studie zur Evaluation der Beratungs- und Koordinationstätigkeit des GPZ. Während Studienteil I die Kostenentwicklung von Eingliederungsleistungen vor und nach Arbeitsaufnahme des GPZ untersucht und gegenüberstellt, wird in Studienteil II die Kosteneffektivität des GPZ prospektiv analysiert: Anhand standardisierter Instrumente (CSSRI, SF-12) wird überprüft, inwiefern sich die Inanspruchnahme von Gesundheitsleistungen und die Lebensqualität der Nutzer durch das Angebot des GPZ verändert hat.

In dem hier behandelten Studienteil III wurden folgende Forschungsfragen behandelt: 1 . Welche Erfahrungen haben die Nutzer mit der Beratung im GPZ gemacht? 2. Wie zufrieden sind sie mit der Beratung?

\section{Material und Methoden}

Zur Erhebung der Patientenerfahrungen wurden offene, leitfadengestützte Einzelinterviews gewählt [17]. Der Leitfaden wurde in Zusammenarbeit mit dem Personal des GPZ entwickelt. Im Vorfeld wurde eine (internationale) Literaturrecherche mit Schlüsselwörtern (Patientenerfahrungen und -zufriedenheit, gemeinsame Entscheidungsfindung, psychosoziale Beratung, Krisendienst) und nach dem Schneeballprinzip durchgeführt. Die Ergebnisse wurden bei der Konstruktion des Leitfadens verwendet. Folgende Schwerpunkte wurden gesetzt:

- Anlass und Inhalte der Beratung,

- positive und negative Erfahrungen,

- Beziehung zwischen Klient und Berater,

- Entscheidungsfindung,

- Ergebnisse der Beratung.

\section{Stichprobenrekrutierung und -beschreibung}

Es erfolgte ein Convenience Sampling. Die Rekrutierung der Teilnehmer wurde durch das GPZ-Personal vor Ort vorgenommen. Die Nutzer wurden jeweils im Nachgang der Beratung auf ihr Interesse zur Studienteilnahme angesprochen. Im Durchschnitt lagen fünf Tage (minimal zwei, höchstens acht Tage) zwischen Beratungstermin und Interview.

Eingeschlossen wurden mindestens 18 Jahre alte Nutzer mit mindestens zwei wahrgenommenen Beratungen. Ausgeschlossen wurden Personen mit unzureichenden Deutschkenntnissen sowie einer akuten oder mit erheblichen kognitiven Defiziten einhergehenden psychischen Störung. Alle Teilnehmer gaben ihre informierte schriftliche Zustimmung für die Studienteilnahme.

Die Studie wurde von der Ethikkommission der Ärztekammer Schleswig-Holstein bewilligt (Az. 131/14).

\section{Datenerhebung und Auswertung}

Die Datenerhebung erfolgte zweiphasig im November 2014 und Februar 2015. Alle Interviews wurden vom Erstautor (JS) geführt, digital aufgezeichnet und anonymisiert transkribiert. Als Methode zur Analyse 
wurde die zusammenfassende qualitative Inhaltsanalyse nach Mayring gewählt [18]. Ziel war es, das Material in eine kompaktere Form zu überführen, die noch immer Abbild des Grundmaterials ist. Erhebung und Analyse fanden abwechselnd statt, bis keine neuen Erkenntnisse mehr erzielt wurden, also "theoretische Sättigung" eintrat [19]. In einem alternierend deduktiv-induktiven Prozess entstand ein Kategoriensystem aus Haupt- und Unterkategorien (vgl. Abb. 2). Um substanzielle Gütekriterien qualitativer Forschung zu berücksichtigen, wurden die Transkripte konsensuell kodiert. Der Fortgang von Analyse und Auswertung wurde regelmäßig im Forschungsteam (JS, BB, BS und AB) diskutiert.

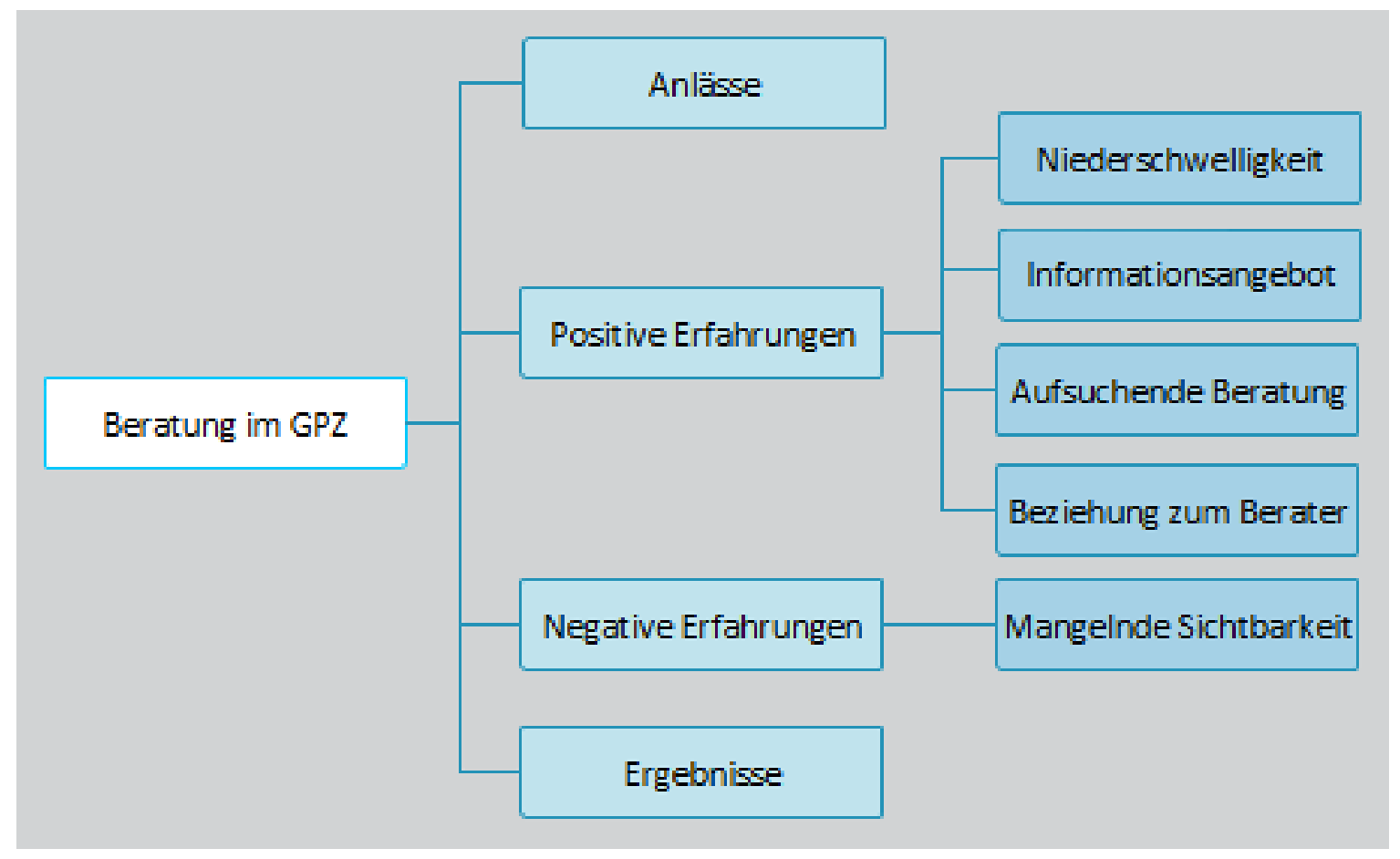

Abb. 2 Beratung im GPZ - Haupt- und Unterkategorien im Überblick.

\section{Ergebnisse}

Insgesamt wurden 13 Personen angefragt, wobei vier die Teilnahme - ohne Angabe von Gründen im Vorfeld ablehnten (Response-Rate $=69,2 \%$ ). Tab. 2 zeigt die soziodemographischen Eigenschaften der Stichprobe $(n=9)$. 
Tab. 2 Soziodemographie der Nutzer $(n=9)$.

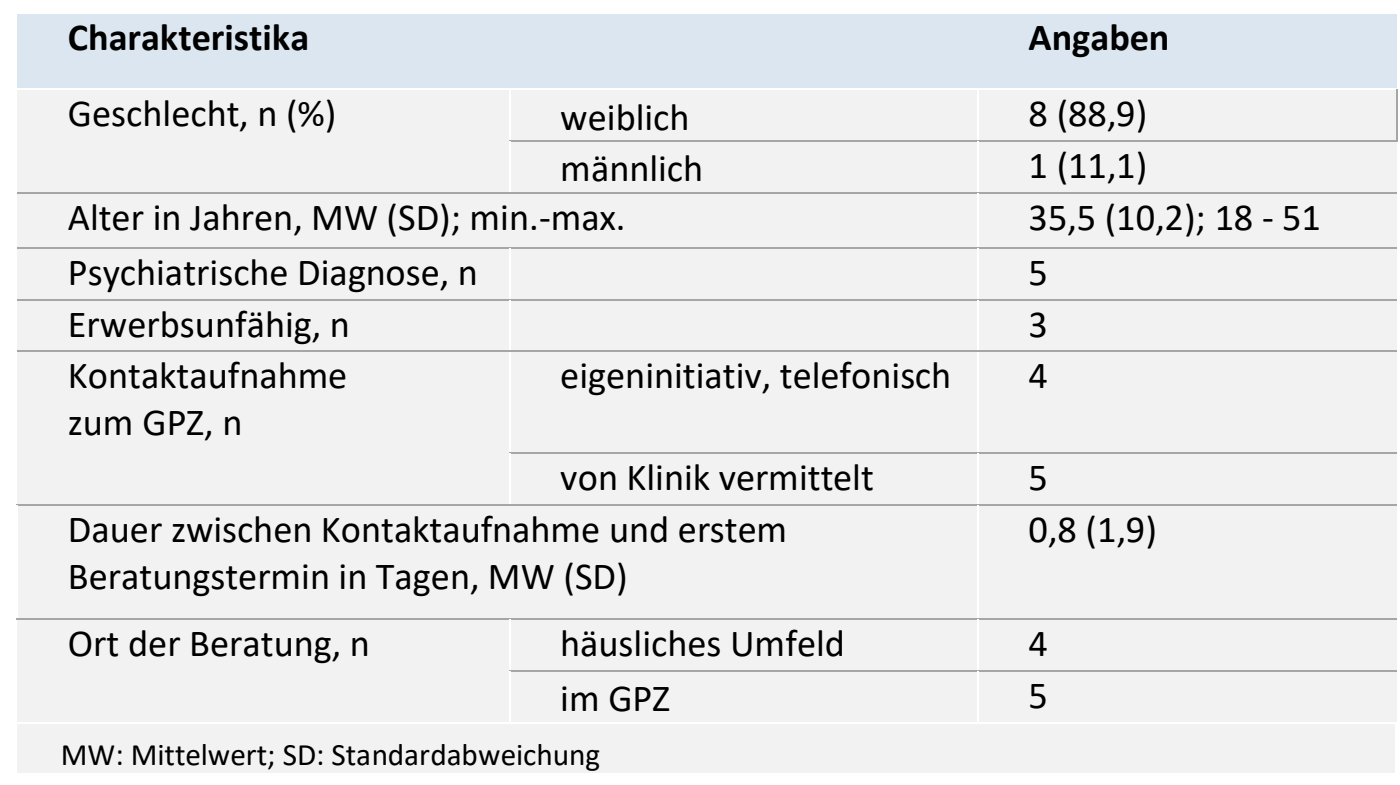

Der Erstautor führte ein Probeinterview (Pretest) durch. Dieses konnte in der Stichprobe berücksichtigt werden, da sich am Leitfaden kein Überarbeitungsbedarf zeigte. Die durchschnittliche Dauer betrug 21 Minuten.

\section{Kategoriale Darstellung}

Im Zentrum der Ergebnisse stehen die Erfahrungen mit der Beratung im GPZ. Abb. 2 zeigt das hierzu entwickelte Kategoriensystem. Den Hauptkategorien „Anlässe“ und „Ergebnisse“ sind ebenfalls Unterkategorien zugeordnet (vgl. Tab. 3 und Tab. 4), die aus Gründen der Übersichtlichkeit nicht in der Abbildung dargestellt sind.

\section{Beratungsanlässe}

Die Studienteilnehmer stellten sich mit einer Reihe unterschiedlicher Probleme im GPZ vor und gaben mehrheitlich an, sich in einer psychosozialen Krise zu befinden. Tab. 3 zeigt anhand exemplarischer Äußerungen die Vielfalt der Beratungsanlässe auf. Neben üblichen Themen wie Überforderung in verschiedenen Lebensbereichen, scheint das GPZ auch eine Anlaufstelle für Patienten zu sein, die negative Erfahrungen mit stationären Aufenthalten gemacht haben, solche ablehnten, oder nicht krankenversichert waren. Die Zitate sind mit einem Kürzel versehen, der auf den Nutzer verweist, von dem die jeweilige Äußerung stammt. 
Tab. 3 Unterkategorien der Hauptkategorie „Anlässe“ der Beratung mit Beispielzitaten.

Unterkategorien
Beziehungskonflikte
Familiäre oder berufliche
Probleme
Psychosoziale Notlage
Einschätzung Hilfebedarf
Nachstationäre
Versorgung
Verhinderung stationärer
Aufnahme

\section{Textbeispiel}

"Es war eben halt so gewesen, dass meine Frau irgendwann zu mir gesagt hat, dass sie sich scheiden lassen wollte, ich noch eine ganze Zeit lang zu Hause versucht habe, damit klar zu kommen und als das dann irgendwann nicht mehr ging, bin ich an die Beraterin herangetreten" (N4).

"Der Anlass war, dass ich wieder in eine Depression gefallen bin [...], weil meine Mutter vor einem halben Jahr einen Schlaganfall hatte und ich deswegen jetzt gezwungen bin, alleine zu wohnen" (N3).

„Meine Lebenskrise wurde verursacht durch eine Insolvenz beruflicher Art. Ich war selbstständig gewesen. [...] Es war aber einfach so, dass ich sehr sehr starke Schuldgefühle gegenüber den Gläubigern hatte, [...] das war wirklich so der Hauptpunkt" (N5). „Ja also ich stell mir halt vor, ja dass die Beraterin sich das anhört und mir dann [...] Therapeuten empfieh/t. Entweder sagt sie, ja eigentlich hast Du gar nichts, das hat nichts mit früher zu tun, oder, dass man doch sieht, ok, das hat was damit zu tun und möchte halt mit einem Therapeuten darüber sprechen" (N6).

"Als ich aus der Klinik nach Hause kam, stürzte halt auch alles wieder auf mich ein. Es war ganz viel mit meinem Sohn zu regeln, das hat mich einfach alles überfordert" (N9). "Also es war immer so ein, was ist jetzt eigentlich im Vordergrund, so. Klinik haben wir überlegt, da habe ich gesagt, [...] mache ich nicht, will ich nicht!" (N7).

Tab. 4 Unterkategorien der Hauptkategorie „Ergebnisse“ der Beratung mit Beispielzitaten.

\begin{tabular}{|c|c|}
\hline Unterkategorien & Textbeispiel \\
\hline Emotionale Entlastung & $\begin{array}{l}\text { "Also mich bringt's auf jeden Fall weiter. Und ich merke auch wie ich peu-a-peu - wie } \\
\text { gesagt - von meinem ganzen Druck den ich innerlich hatte - runter komme, der sich so } \\
\text { ein bisschen löst und auch sagen kann - heute ist einfach mal so" (N8). }\end{array}$ \\
\hline Psychoedukation & $\begin{array}{l}\text { „Ja, also dass wir jetzt einen Plan gemacht haben, was ich zuhause mit dem Freund } \\
\text { verändern kann, da habe ich eben halt Tipps bekommen, wie man das ändern kann } \\
\text { [...]. Also, das hat schon wirklich viel gebracht" (N6). }\end{array}$ \\
\hline $\begin{array}{l}\text { Ressourcenorientierte Be- } \\
\text { darfseinschätzung }\end{array}$ & $\begin{array}{l}\text { "Also die Beraterin war der Meinung, dass ich eine so starke rechtliche Betreuung } \\
\text { nicht brauche, weil ich ja so eigentlich noch geistig doch auf der Höhe bin [...] und das } \\
\text { alles ganz gut einschätzen kann." (N1) }\end{array}$ \\
\hline Qualifizierte Vermittlung & $\begin{array}{l}\text { "Und hier über das GPZ habe ich dann glücklicherweise jetzt eine Psychologin gefun- } \\
\text { den, die ist ungefähr } 30 \text { Kilometer weit weg, aber sie hat nächste Woche schon einen } \\
\text { Termin für mich" (N9). }\end{array}$ \\
\hline $\begin{array}{l}\text { Überbrückung von Warte- } \\
\text { zeiten }\end{array}$ & $\begin{array}{l}\text { "Ich suche aber immer noch Hilfe im GPZ [...], weil ich sollte in ambulante Psychothera- } \\
\text { pie, das geht aber vor Dezember nicht, weil da immer alles besetzt ist" (N4). }\end{array}$ \\
\hline $\begin{array}{l}\text { Lösung von Behördenan- } \\
\text { gelegenheiten }\end{array}$ & $\begin{array}{l}\text { "Und die Beraterin hat mir auch dabei geholfen, [...] dass ich die Betreuerin vom Amts- } \\
\text { gericht schneller bekomme. Sie hat ein bisschen Druck gemacht, weil ich habe jeman- } \\
\text { den gebraucht, der wirklich alle Behördenangelegenheiten für mich übernehmen } \\
\text { kann." (N3) }\end{array}$ \\
\hline Einbezug Angehöriger & $\begin{array}{l}\text { "Also das Ergebnis war, das ich mich auch mit meiner Freundin hingesetzt habe, nach } \\
\text { dem Gespräch. Die Beraterin hatte auch mit ihr gesprochen [...], weil sie auch wissen } \\
\text { wollte, wie ich mich gemacht habe, seitdem ich aus der Klinik bin" (N8). }\end{array}$ \\
\hline
\end{tabular}

\section{Erfahrungen mit der Beratung}

\section{Niederschwelligkeit}

Besonderen Stellenwert unter den positiven Erfahrungen nimmt die Niederschwelligkeit ein. Die Tatsache, dass die Beratung im Rahmen eines GPZ und nicht als Klinikleistung angeboten wurde, führte zu einem Gefühl von Autonomie und Unabhängigkeit von der psychiatrischen Klinik. 
„ICh hatte Angst alleine rauszukommen aus dem Krankenhaus, ohne Unterstützung. Aber jetzt so

- Sicherheit. Einfach die Sicherheit, dass die Beraterin da ist, das macht schon viel aus" (N8).

In diesem Zusammenhang wurde die entlastende Wirkung der telefonischen Rufbereitschaft betont.

„Die Beraterin hat mir die Telefonnummer gegeben, ich kann sie auch jederzeit anrufen. Also von daher fühle ich mich sicherer, dass es so etwas gibt" (N8).

Fünf der neun Befragten waren besonders zufrieden mit der zeitnahen Terminvergabe und den kurzen Wartezeiten.

„Die Beraterin hat sofort einen Termin mit mir gemacht, für den nächsten Tag, da war ich echt froh" (N7).

Ferner begrüßten die Klienten, dass die Beratung auch ohne Krankenversicherung unentgeltlich angeboten wird. Eine Nutzerin war beispielsweise zum Zeitpunkt der Inanspruchnahme der Beratung nur im GKV/PKV-Notlagentarif versichert.

„Ein sehr großer Vorteil war die kostenlose Beratung - ich habe aufgrund der Insolvenz [...] große Probleme mit der Krankenkasse [...]. Und eine stationäre Aufnahme in eine Klinik wäre für mich sowohl privat als auch eben finanziell überhaupt nicht möglich, weil ich nicht wusste, ob ich überhaupt abgesichert bin" (N5).

\section{Informationsangebot}

Es wurden häufig Informationen über Begegnungs- und Tagesstätten, Selbsthilfegruppen und andere gemeindepsychiatrische Hilfen vermittelt. Die Befragten waren sowohl mit der Qualität als auch dem Umfang des Informationsangebots zufrieden und gaben an, dass die Informationen, die sie erhalten hatten, ihren Bedürfnissen entsprachen.

„Aber das finde ich wichtig, dass die Beraterin auch gleich wusste, dass es da eine Selbsthilfegruppe für die Behinderung meines Kindes gibt" (N9).

\section{Aufsuchende Beratung}

Insbesondere Nutzer mit sozialen Rückzugstendenzen oder körperlichen Einschränkungen schienen von der aufsuchenden Beratung zu profitieren.

„Ja, nach Hause kommen, das ist vielleicht ein bisschen leichter, weil [...] ich traue mich ja sowieso meistens nicht raus, weil ich dann viele Leute nicht kenne und dann ziehe ich mich zurück" (N2).

„Ich habe derzeit auch noch eine Verbrennung am Fuß, da kam wirklich alles zusammen. Die Beraterin hat sofort gesagt: Ja, dann komme ich gerne zu ihnen" (N7).

Das GPZ-Personal könne sich vor Ort ein besseres Bild von den Lebensumständen der Klienten machen und sich so besser einfühlen. Darüber hinaus falle es Nutzern im gewohnten Umfeld leichter, sich zu äuBern.

"Ich finde, diese Hausbesuche persönlicher, wenn man sich gegenübersitzt und miteinander spricht, [...] in einem normalen Umfeld. Und dass sich die Beraterin dann auch so besser in mich 
reinversetzen kann. Ich finde das Krankenhaus ist dann immer so ein bisschen nicht steril, aber das ist so ein bisschen befremdlich. Und zu Hause ist das [...] anders. Also, da kommt man dann, wenn es einem schlecht geht [...] auch mehr aus sich raus" (N1).

Besonders in Krisensituationen schien die aufsuchende Beratung hilfreich, da der Erhalt des Umfeldes und der Tagesstruktur auf Betroffene stabilisierend wirke. Schließlich sei auch die Hürde deutlich geringer, die Beratungsleistungen anstelle des Notdienstes der pflichtversorgenden psychiatrischen Klinik in Anspruch zu nehmen. Die Tatsache, dass die Berater ihre Klienten zuhause besuchten, wurde als wertschätzend erlebt.

„Und ich hab das so gesehen wie die Beraterin meine häusliche Umgebung wahrgenommen hat [...]. Das habe ich nicht vergessen. Gut, jeder ist anders, aber für mich war das wichtig. Diese Wertschätzung, so. Für mich als Person, wo ich lebe. [...] Und nach dem Gespräch habe ich gedacht, ich bin doch noch ein normaler Mensch" (N7).

\section{Beziehung zum Berater}

Aus Sicht der Befragten spielte die ihnen entgegengebrachte Empathie und Wertschätzung eine wichtige Rolle für die Güte der Beziehung zwischen Nutzer und Berater. Dabei ähnle der Kontakt zum Berater weniger einer ärztlichen Beziehung, die von den Nutzern als ",objektiv", „formal" und "distanziert" charakterisiert wurde. Vielmehr zeichne sich das Verhältnis durch die Kombination freundschaftlich-familiärer und professioneller Anteile aus.

„Es war schon professionell, finde ich. Aber es war nicht, vom Gefühl her, dieses typische, wie wenn man zum Arzt geht, dass da alles exakt fachlich beredet wurde. Also es war schon ein klein wenig auf dieser Ebene, wie mit einer Freundin, aber es war trotzdem eben professionell" (N3).

Im Gespräch getroffene Entscheidungen wurden in einem gemeinsamen Prozess zwischen Klient und Berater ausgehandelt. Ein häufiger Diskussionspunkt war der gemeinsame Umgang mit der Ablehnung einer nahegelegten stationären Aufnahme.

„Diese typische Angst im Bekanntenkreis [...], du bist depressiv, du kriegst sofort Medikamente und wirst eingewiesen. Also was ich sehr schön fand - es wurde mit mir gesprochen, ob ich das möchte, ob ich wirklich mal vier Wochen stationär aufgenommen werden möchte. Als ich gesagt habe, das möcht ich nicht, gab es auch die Möglichkeit eine leichte Medikation über den Hausarzt zu machen [...]. Und ich fand es sehr schön, dass ich die Wahl hatte und das für mich Stimmige rausfiltern konnte" (N5).

\section{Mangelnde Sichtbarkeit der Beratungsstelle}

Einzelne Teilnehmer äußerten sich kritisch über die fehlende Sichtbarkeit des GPZ in den gängigen Suchmaschinen und bei potentiell zuweisenden Akteuren wie dem Jobcenter oder sonstigen Beratungsstellen. 
Die nachfolgende Äußerung stammt von einer Klientin, die bereits mehrere Monate vergebens nach Hilfe gesucht hatte, bevor sie an das GPZ vermittelt wurde.

„Ich finde einfach, dass diese Angebote deutlich mehr präsent sein müssen bei Institutionen, die sich z.B. mit Arbeitslosen, Insolvenzen - es gibt ja so viele andere Situationen wo man in Stress, in Notsituationen gerät - befassen. Dass es einfach viel verbreiteter sein müsste, weil ich ewig gebraucht habe, bevor ich tatsächlich eine Anlaufstelle hatte. Also das finde ich, ist einer der ganz ganz wichtigen Punkte" (N5).

\section{Ergebnisse der Beratung}

Zunächst führte die Möglichkeit, die psychosozialen Probleme artikulieren zu können, bei den Befragten zu einer deutlichen emotionalen Entlastung (vgl. Tab. 4). Häufig konnte ein umfassenderes Problem- bzw. Störungsverständnis bei den Klienten oder ihren Angehörigen erreicht und Strategien zum Umgang mit den Problemen entwickelt werden. Klienten, die ihren individuellen Hilfebedarf nicht einschätzen konnten, wurden unterstützt, diesen zu ermitteln. Hierzu wurden geeignete, ressourcenorientierte Hilfsmaßnahmen aufgezeigt und Fehleinschätzungen über die Angemessenheit bestimmter Interventionen konnten korrigiert werden. Ein wichtiges Ergebnis aus Nutzersicht ist die qualifizierte Vermittlung an einen Psychotherapeuten. So konnten Nutzer, die im Vorfeld vergebens nach einem Therapieplatz gesucht hatten, z. T. ohne Wartezeit in ein Erstgespräch vermittelt werden. In anderen Fällen konnte die Wartezeit auf einen Psychotherapieplatz durch die Beratung überbrückt werden.

\section{Diskussion}

Mit ihren überwiegend positiven Äußerungen gaben die Befragten einen umfassenden Einblick in die Ursachen ihrer Zufriedenheit mit den Beratungs- und Koordinationsleistungen des GPZ in Heide.

Die Tatsache, dass die Beratung meistens am Tag der Kontaktaufnahme oder am Folgetag in Anspruch genommen wurde, spricht für die Dringlichkeit der Anliegen und deutet auf die Gewährleistung eines zeitnahen Beratungsgesprächs hin. Aus den Beratungsanlässen wird deutlich, dass ein Großteil der Befragten soziale Problemlagen für die Entstehung der jeweiligen Krisensituation verantwortlich machte, während psychische Störungen nur am Rande erwähnt wurden. Dennoch scheint das GPZ eine wichtige Rolle als Anlaufstelle für Personen mit psychischen Störungen zu spielen, die nicht stationär behandelt werden möchten. Internationale Studien belegen, dass Nutzer eine Krankenhausbehandlung vor allem dann ablehnen, wenn sie negative Vorerfahrungen im Krankenhaus gemacht haben, oder sich vor „Psychiatrisierung" und Stigmatisierung fürchten $[14,20]$. Die Beratungsstelle des GPZ könnte möglicherweise dabei behilflich sein, einen Zugang zu dieser Gruppe von Patienten anzubahnen. Dies wird unterstützt durch die Aussagen der Nutzer, die nach Inanspruchnahme der Beratung bereit waren, eine stationäre Behandlung in Erwägung zu ziehen. 
Die zahlreichen Aussagen der Studienteilnehmer, die eine Zufriedenheit mit den erhaltenen Leistungen ausdrücken, legen eine starke Ausrichtung des GPZ an den Bedürfnissen und Erwartungen seiner Nutzer nahe [5]. Hierzu gehören vor allem Struktur- und Leistungsmerkmale, die es Ratsuchenden ermöglichen, das Beratungsangebot unter verhältnismäßig geringem Aufwand in Anspruch zu nehmen. So scheint bereits das Wissen um die 24-stündige Verfügbarkeit zu einem Gefühl von Sicherheit und Entlastung zu führen [16,21]. Dies wird begünstigt durch die Flexibilität der Leistungen: Nutzer möchten wählen können, wann, wo und wie lange sie die Hilfe eines Experten in Anspruch nehmen [22]. Den Teilnehmern war es beispielweise wichtig, frei entscheiden zu können, ob sie die Beratung telefonisch, im Zuge eines Hausbesuches oder durch persönliche Vorstellung im GPZ beanspruchten.

In den Äußerungen der Nutzer nimmt das Thema „aufsuchende Beratung“ großen Stellenwert ein. Während für diese Unterstützungsform bisher kaum Evidenz vorliegt, ist belegt, dass stationsersetzende psychiatrische Behandlungssettings gegenüber der Regelbehandlung zahlreiche Vorteile aufweisen. Hierzu gehören u. a. eine höhere Patientenzufriedenheit und langfristige Verbesserung und Stabilisierung des Gesundheitszustandes [23,24]. Abgesehen von der fraglichen Übertragbarkeit dieser Resultate auf Beratungskontexte, legen die Erfahrungen unserer Studienteilnehmer zumindest eine hohe Zufriedenheit mit aufsuchender Beratung nahe. Dies könnte möglicherweise mit der Wertschätzung zusammenhängen, die durch einen Hausbesuch vermittelt wird: Der Nutzer nimmt so nicht die Rolle eines Patienten ein, der eine Institution aufsuchen und warten muss, bis er an der Reihe ist, sondern das Beratungspersonal übernimmt diesen Aufwand für ihn. Ein weiterer potentiell zufriedenheitsbildender Aspekt liegt vermutlich in der alltagsweltlichen Situierung der Leistungsform. So fühlen sich Betroffene in ihrem Lebensumfeld eher als „normaler Mensch“ (vgl. Zitat N7) anstatt als „Patient“ wahrgenommen [21]. Bei den Nutzern des Heider GPZ hatte dies zur Folge, dass sie sich den Beratern gegenüber besser öffnen bzw. mitteilen konnten und sich weniger stigmatisiert fühlten. In der Studie von Jürgensen et al. hingegen nahmen einzelne Patienten eine Gegenposition ein, da sie den Hausbesuch durch die ambulante psychiatrische Pflege als Eingriff in ihre Privatsphäre erlebten [16]. Aufsuchende Unterstützungsformen scheinen folglich vor allem dann angebracht, wenn sie neben der fachlichen Indikation auch von den Leistungsempfängern als hilfreich erachtet werden.

Im Sinne der Patientenorientierung kritisch zu diskutieren ist die Äußerung einer Befragten über die unzureichende Sichtbarkeit des GPZ und das fehlende Wissen von potentiell zuweisenden Akteuren über die Beratungsstelle. Dies hatte für einzelne Nutzer eine verlängerte Hilfesuche zur Folge. Zu ähnlichen Befunden kamen Tanskanen et al., die das Hilfesuchverhalten aus Betroffenensicht untersuchten [25]. So mangelte es insbesondere bei nicht gesundheitsbezogenen kommunalen Anlaufstellen (z. B. dem Jobcenter) oft an Zeit, Wissen und Skills, um beurteilen zu können, wie Personen mit psychischen Problemen angemessen unterstützt und qualifiziert weitervermittelt werden können. Tanskanen et al. schlugen vor, Mitarbeiter von Bildungs- und Gemeindeorganisationen gezielt zu schulen [25]. Da sich das GPZ zum Zeit- 
punkt der Erhebung noch in einer Einführungsphase befand und nur über eine geringe Personalausstattung verfügte, wurde zunächst gezielt auf Schulungsmaßnahmen und Öffentlichkeitsarbeit verzichtet, um die Nachfrage bewältigen zu können, was den Aspekt der fehlenden Sichtbarkeit relativiert.

Neben den Aussagen zur Zufriedenheit mit strukturellen Eigenschaften des GPZ, betonten die Befragten die Bedeutung einer guten Beziehung zum Berater für ihre Zufriedenheit. Es ist allgemein bekannt, dass die sozioemotionale Kompetenz und die Qualität der Beziehung des medizinischen Personals von überragender Bedeutung für die Patientenzufriedenheit sind [10]. An dieser Stelle ist auf die Unterschiedlichkeit von Beratungs- und Behandlungskontexten hinzuweisen, sodass eine Übertragbarkeit von Forschungsergebnissen nur begrenzt möglich ist. Dennoch decken sich die Äußerungen der Beratenden bzw. Befragten der vorliegenden Studie an vielen Stellen mit den Äußerungen von Patienten aufsuchender psychiatrischer Dienste [21,22]. Die Ergebnisse deuten darauf hin, dass es den Nutzern leichter fiel, sich mitzuteilen, wenn sie einen Kontakt auf Augenhöhe anstelle einer paternalistisch geprägten Beziehung zum Berater erfuhren. Die Einbeziehung der Nutzer in Entscheidungen, die in der Beratung getroffen wurden, wirkte sich zudem positiv auf die Beziehungsqualität und die Zufriedenheit mit der Beratung aus [26].

Der generelle Wunsch nach mehr Mitbestimmungsrechten bei gesundheitsbezogenen Entscheidungen spiegelte sich auch in den Äußerungen unserer Studienteilnehmer wider $[15,27]$. Unter den verschiedenen Partizipationsmodellen scheint das Vorgehen im GPZ am ehesten dem der Partizipativen Entscheidungsfindung (PEF) oder der informierten Einwilligung zu entsprechen. Auf einer Skala von „keiner Partizipation“ (Entscheidungsmacht vollständig beim Berater) bis hin zu „informed choice“ (Berater informiert und Klient entscheidet) nimmt PEF eine Mittelstellung ein. Die Äußerungen der Nutzer belegen, dass die Kriterien von PEF nach Charles et al. erfüllt sind [28]. Demnach sind an einer Entscheidung erstens Berater und Beratender beteiligt, zweitens teilen beide Akteure Informationen und drittens führt ein stufenweiser Prozess zu einem Konsens bezüglich des Weiteren Vorgehens. In Anbetracht der Tatsache, dass in der Beratung keine therapeutischen Entscheidungen getroffen, sondern lediglich Handlungsoptionen aufgezeigt werden, ist anzunehmen, dass bei einem Großteil der Sitzungen die Prinzipien der Informierten Einwilligung zugrunde gelegt wurden. Dies wäre wünschenswert, da so den Nutzern ein hoher Grad an Partizipation eingeräumt würde.

\section{Limitationen}

Bedingt durch die Heterogenität gemeindepsychiatrischer Beratungsstellen in Verbindung mit den strukturellen Besonderheiten des Heider GPZ (z. B. rechtskreisübergreifende Ausrichtung), sind die Ergebnisse nur eingeschränkt generalisierbar. In den demographischen Daten (Abb. 2) fällt der äußerst hohe Anteil weiblicher Befragter (ca. 90\%) ins Auge. Unveröffentlichte statistische Leistungsdaten des GPZ bestätigen die deutliche Verschiebung in Richtung des weiblichen Geschlechts. Dies deckt sich mit der Tatsache, dass Frauen im Allgemeinen ihre Beschwerden selbst besser wahrnehmen als Männer, bereitwilliger darüber berichten und schneller Hilfe in Anspruch nehmen [29]. Darüber hinaus belegen empirische Befunde, dass 
Frauen sich in Umfragen zur Patientenzufriedenheit doppelt so häufig wie Männer nicht trauen, sich zu beschweren [30]. Dies könnte eine der Ursachen sein, weshalb in der vorliegenden Studie nur sehr wenig negative Erfahrungen mitgeteilt wurden. Da das GPZ sich in vielen Aspekten an den Bedürfnissen der Nutzer orientiert und mit seinem Angebot eine tatsächliche Versorgungslücke schließt, erscheinen die überwiegend positiven Erfahrungen jedoch nachvollziehbar. Weiterhin fällt auf, dass sich nicht unmittelbar über die Vorteile der rechtskreisübergreifenden Funktion geäußert wurde. Dies ist einerseits verständlich, handelt es sich um eine „technische“ Eigenschaft des Angebots, die konkrete Nutzer vermutlich nicht interessiert. Andererseits finden sich in den Ergebnissen (Tab. 4) einige implizite Hinweise, dass von der rechtskreisübergreifenden Ausrichtung profitiert wurde: Die Nutzer gaben an, dass ihnen sowohl bei psychischen als auch sozialen Anliegen (z. B. rechtliche Betreuung, Behördenangelegenheiten) umfassend geholfen werden konnte.

\section{Fazit für die Praxis}

- Das Vorhandensein einer rund-um-die-Uhr verfügbaren, psychosozialen Beratungsstelle erhöht bei Nutzern das Gefühl von Sicherheit und Autonomie.

- Durch aufsuchende Beratung lässt sich ein Kontakt zu Betroffenen anbahnen, die Hilfe benötigen, aber das gegenwärtige Hilfesystem nicht in Anspruch nehmen möchten, oder dies störungsbedingt nicht können.

- Neben den strukturellen Aspekten wirken sich auch die Qualität der Beratungsbeziehung sowie die Art der Entscheidungsfindung im Gespräch auf die Bewertung durch die Nutzer aus.

\section{Danksagung}

Die Autoren danken den Ansprechpartnern des GPZ - Torsten Hejnal und Frank Röwenstrunk - für ihre Unterstützung und allen Nutzern für ihre Offenheit und die konstruktiven Gespräche.

\section{Förderung}

Die Studie wurde durch das Land Schleswig-Holstein finanziert (Fördernummer: 234-455.5.004-005/02).

\section{Interessenskonflikt}

Die Autoren geben an, dass kein Interessenskonflikt besteht. 


\section{Literatur}

${ }^{1}$ Deutscher Bundestag. Gesetz zur Stärkung der Teilhabe und Selbstbestimmung von Menschen mit Behinderungen. Im Internet: www.bmas.de/SharedDocs/Downloads/DE/PDF-Meldungen/2016/bundesteilhabegesetz.pdf

2 Längle G, Gottlob M, Elsässer-Gaißmaier H-P. Umsetzung des § 115d PsychVVG: Stationsäquivalente Behandlung - Die nächsten Schritte. Psychiatr Prax 2017; 44: 417-419

${ }^{3}$ Steinhart I, Wienberg G. Das Funktionale Basismodell für die gemeindepsychiatrische Versorgung schwer psychisch kranker Menschen - Mindeststandard für Behandlung und Teilhabe. Psychiatr Prax 2016; 43: 65-68

${ }^{4}$ Steinhart I, Wienberg G (Hrsg.). Rundum ambulant. Funktionales Basismodell psychiatrischer Versorgung in der Gemeinde. Köln: Psychiatrie Verlag, 2017

${ }^{5}$ Biringer E, Davidson L, Sundfør B, Ruud T, Borg M. Service users' expectations of treatment and support at the Community Mental Health Centre in their recovery. Scand J Caring Sci 2016; 31:505-513

${ }^{6}$ Attkisson CC, Zwick R. The Client Satisfaction Questionnaire: Psychometric properties and correlations with service utilization and psychotherapy outcome. Eval Program Plann 1982; 5: 233-237

${ }^{7}$ Schmidt J, Lamprecht F, Wittmann WW. Zufriedenheit mit der stationären Versorgung. Entwicklung eines Fragebogens und erste Validitätsuntersuchungen. Psychother Psychosom Med Psychol 1989; 39: 248-255

${ }^{8}$ Rentrop M, Böhm A, Kissling W. Patientenzufriedenheit mit stationär psychiatrischer Behandlung. Fortschritte Neurol · Psychiatr 1999; 67: 456-465

${ }^{9}$ Jacob G, Bengel J. Das Konstrukt Patientenzufriedenheit: Eine kritische Bestandsaufnahme. Z Für Klin Psychol Psychiatr Psychother 2000; 48: 280-301

${ }^{10}$ Giupponi G, Hensel S, Müller P, Soelva M, Schweigkofler H, Steiner E, Pycha R, Möller-Leimkühler A. Behandlungszufriedenheit und Einflussfaktoren in der stationären Psychiatrie: ein deutsch/italienischer Vergleich. Fortschritte Neurol · Psychiatr 2009; 77: 346-352

${ }^{11}$ SpießI H, Leber C, Radlinger P, Schmid R, Kaatz S, Binder H, Hambrecht M, Klein HE, et al. Patientenzufriedenheit in psychiatrischen Kliniken. Nervenheilkunde 2009; 28: 481-486

${ }^{12}$ Spill B, Beitinger R, Kissling W, Hamann J. Patientenzufriedenheit mit einem Programm der Integrierten Versorgung für die Indikationen Schizophrenie und Depression. Psychiatr Prax 2013; 40: 142-145

${ }^{13}$ Manary MP, Boulding W, Staelin R, Glickman SW. The Patient Experience and Health Outcomes. N Engl J Med 2013; 368: 201-203

${ }^{14}$ Newman D, O'Reilly P, Lee SH, Kennedy C. Mental health service users' experiences of mental health care: an integrative literature review. J Psychiatr Ment Health Nurs 2015; 22: 171-182

${ }^{15}$ Elstad TA, Eide AH. User participation in community mental health services: exploring the experiences of users and professionals. Scand J Caring Sci 2009; 23: 674-681

${ }^{16}$ Jürgensen M, Patzelt C, Meyer T. „Dass man also frei bleibt, aber immer das Gefühl hat: Da ist diese Haltestange, die ich brauch“. Psychiatr Prax 2014; 41: 29-36

${ }^{17}$ Flick U. » Das halbstandardisierte Interview «. In: Flick U (Hrsg.). Qualitative Sozialforschung. Eine Einführung. Reinbek: Rowohlts, 2012: 203-209 
${ }^{18}$ Mayring P. Qualitative Inhaltsanalyse: Grundlagen und Techniken. 12. Aufl. Weinheim: Beltz, 2015

${ }^{19}$ Strauss A, Corbin JM. Grounded theory in practice. Sage, 1997

${ }^{20}$ Sercu C, Bracke P. Stigma, Social Structure, and the Biomedical Framework: Exploring the Stigma Experiences of Inpatient Service Users in Two Belgian Psychiatric Hospitals. Qual Health Res 2016; 27:12491261

${ }^{21}$ Winness MG, Borg M, Kim HS. Service users' experiences with help and support from crisis resolution teams. A literature review. J Ment Health 2010; 19: 75-87

${ }^{22}$ Hopkins C, Niemiec S. Mental health crisis at home: service user perspectives on what helps and what hinders. J Psychiatr Ment Health Nurs 2007; 14: 310-318

${ }^{23}$ Gühne U, Weinmann S, Arnold K, Atav E-S, Becker T, Riedel-Heller S. Akutbehandlung im häuslichen Umfeld: Systematische Übersicht und Implementierungsstand in Deutschland. Psychiatr Prax 2011; 38: 114-122

${ }^{24}$ Lambert M, Schöttle D, Ruppelt F, Lüdecke D, Sarikaya G, Schulte-Markwort M, Gallinat J, Karow A. Integrierte Versorgung für erst- und mehrfacherkrankte Patienten mit schweren psychotischen Erkrankungen: 3-Jahres-Ergebnisse des Hamburger Modells. Bundesgesundheitsblatt - Gesundheitsforschung - Gesundheitsschutz 2015; 58: 408-419

${ }^{25}$ Tanskanen S, Morant N, Hinton M, Lloyd-Evans B, Crosby M, Killaspy H, Raine R, Pilling S, Johnson S. Service user and carer experiences of seeking help for a first episode of psychosis: a UK qualitative study. BMC Psychiatry 2011; 11: 157

${ }^{26}$ Hamann J, Loh A, Kasper J, Neuner B, Spies C, Kissling W, Härter M, Heesen C. Partizipative Entscheidungsfindung. Nervenarzt 2006; 77: 1071-1078

${ }^{27}$ Loos S, Neumann P, Arnold K, Slade M, Fiorillo A, Krogsgaard Bording M, Ivanka T, Kawohl W, Puschner B. Gemeinsame Entscheidungsfindung in der Behandlung von Patienten mit schweren psychischen Erkrankungen. Psychiatr Prax 2013; 40: 23-29

${ }^{28}$ Charles C, Gafni A, Whelan T. Shared decision-making in the medical encounter: what does it mean? (or it takes at least two to tango). Soc Sci Med 1997; 44: 681-692

${ }^{29}$ Belz M, Riecher-Rössler A. Geschlechtsspezifische Aspekte in der Psychotherapie. In: Herpertz S, Caspar F, Lieb K (Hrsg.). Psychotherapie. München: Urban \& Fischer Verlag, 2017, S. 553-565

${ }^{30}$ Stahl K. Beschwerde- und Feedbackmanagement. In: Fischer A (Hrsg.). Servicequalität und Patientenzufriedenheit im Krankenhaus: Konzepte, Methoden, Implementierung. MWV, 2015: 177-184 